\title{
An explicit harmonic code for black-hole evolution using excision
}

\author{
Béla Szilágyi $^{1}$, Denis Pollney ${ }^{1}$, Luciano Rezzolla ${ }^{1,2}$, Jonathan Thornburg ${ }^{1}$ \\ and Jeffrey Winicour ${ }^{1,3}$ \\ ${ }^{1}$ Max-Planck-Institut für Gravitationsphysik, Albert-Einstein-Institut, Golm, \\ Germany \\ 2 Department of Physics, Louisiana State University, Baton Rouge, LA, USA \\ ${ }^{3}$ Department of Physics and Astronomy, University of Pittsburgh, Pittsburgh, PA, USA
}

Received 22 December 2006, in final form 22 March 2007

Published 4 June 2007

Online at stacks.iop.org/CQG/24/S275

\begin{abstract}
We describe an explicit in time, finite-difference code designed to simulate black holes by using the excision method. The code is based upon the harmonic formulation of the Einstein equations and incorporates several features regarding the well-posedness and numerical stability of the initialboundary problem for the quasilinear wave equation. After a discussion of the equations solved and of the techniques employed, we present a series of testbeds carried out to validate the code. Such tests range from the evolution of isolated black holes to the head-on collision of two black holes and then to a binary black hole inspiral and merger. Besides assessing the accuracy of the code, the inspiral and merger test has revealed that the marginally trapped surfaces contained within the common apparent horizon of the merged black hole can touch and even intersect. This novel feature in the dynamics of the marginally trapped surfaces is unexpected but consistent with theorems on the properties of these surfaces.
\end{abstract}

PACS numbers: 04.25.Dm, 02.70.-c, 02.70.Bf, 02.60.Lj

(Some figures in this article are in colour only in the electronic version)

\section{Introduction}

The numerical calculation of the inspiral and merger of a binary black hole system attracted early attention because its dynamic and strongly relativistic gravitational fields were expected to play a major role in astrophysics and to provide an excellent arena for studying the fully nonlinear behaviour of gravitation. It has long been known [1] that the final decay, coalescence and ringdown of such a system is a very strong source of gravitational radiation. Because of their complexity and nonlinearity, and the lack of any continuous symmetries, the Einstein 
equations cannot be solved analytically for these systems. Rather, direct numerical integrations must be used.

Building on an earlier attempt by Hahn and Lindquist [2], the first successful numerical simulations of binary black hole systems were performed in the 1970s by Čadež [3], Eppley [4], DeWitt, and their colleagues [5-7]. These simulations were restricted to axisymmetry, and used the Arnowitt-Deser-Misner (ADM) formulation of the Einstein equations [8]. However, the ADM equations are now known to be only weakly hyperbolic [9], so they are not suitable for long-term numerical evolutions despite large-scale efforts [10].

Starting with the first long-term evolutions by Pretorius [11], there has been remarkable progress in the simulation of binary black holes. Several groups have since tracked the inspiral and merger phases using codes solving a conformal-traceless formulation of the Einstein equations and treating the black holes either by the excision method [12] or by the moving-punctures method [13-16]. Since Pretorius' original work, there has also been substantial progress in developing mathematical theorems which establish the well-posedness of the harmonic initial-boundary value problem and the stability of its finite difference approximations. We have incorporated some of this theory in developing an explicit in time, finite-difference harmonic code to treat the excision problem. We present here some promising initial results indicating that the binary black hole merger can be treated using excision with a minimal amount of dissipation and with sufficient accuracy to reveal new and interesting dynamics of the individual marginally outer trapped surfaces (MOTS) contained within the common apparent horizon.

Harmonic coordinates were first introduced by de Donder [17] to reduce Einstein's equations to ten quasilinear wave equations and they were later extensively developed by Fock [18] and used by Choquet-Bruhat [19] to give the first well-posed version of the Cauchy problem for the gravitational field. In a harmonic gauge the spacetime coordinates $x^{\mu}$ (viewed as a set of four scalar functions) satisfy the curved-space wave equation $\nabla_{c} \nabla^{c} x^{\mu}=0$. The principal part of the Einstein equations then reduces to a second-order hyperbolic form or to a first-order symmetric hyperbolic form, for which there is an extensive mathematical and computational literature. Many researchers have since implemented numerical evolution schemes for harmonic formulations of Einstein's equations [11, 20-30] and the related $Z^{4}$ formulation [31, 32].

The AEI harmonic code presented here has its roots in the Abigel code [22, 23, 25], a second-order accurate, finite-difference code which incorporates theorems establishing the well-posedness and numerical stability of the harmonic initial-boundary value problem. In addition, the AEI code incorporates a black-hole excision algorithm which allows for motion of the excised region across the grid, a superluminal evolution algorithm and, except for regions near the boundaries and for some aspects of the mesh refinement, a fourthorder accurate finite-difference approximation. It also utilizes an apparent horizon finder [33, 34], vertex-centred mesh-refinement techniques [35], black hole initial data sets and other features of the Cactus computational toolkit [36] necessary for black hole simulations. A Runge-Kutta integration is used to carry out an explicit time evolution. Note that this differs from the approach of Pretorius who uses a pointwise Newton-Gauss-Seidel relaxation scheme.

Although some aspects of the code are still under development, most notably the excision boundary and the outer boundary treatments, we report here a series of testbeds carried out to validate the code. Such tests range from the evolution of isolated black holes to the head-on collision of two black holes and then to a binary black hole inspiral and merger. While these tests have now become standard, we have found a new feature in our study of the binary inspiral and merger. In particular, after a common apparent horizon has formed, our simulations show 
that the two individual MOTS approach and continue on to intersect each other. This novel feature in the dynamics of the MOTS is unexpected but not unreasonable if these surfaces have to maintain their smoothness as they meet. It is also consistent with recent theorems concerning the properties of MOTS [37].

The paper is organized as follows. In section 2, we present the system of evolution equations and describe its reduction to first-order in time form, as well as our use of gauge conditions and boundary conditions. In section 3, we give a brief discussion of the numerical techniques we have employed. Section 4 collects the results of our tests and the calibration of the code's accuracy. Finally, section 5 summarizes our results and the prospects for future work.

\section{Harmonic evolution system}

\subsection{The evolution system}

Although completely redesigned, the AEI harmonic evolution code is based on the work presented in Babiuc, Szilágyi et al [22, 23, 25] with modifications to allow for a smooth transition from subluminal to superluminal evolution, together with higher-order finitedifference operators and the possibility of excising an arbitrary portion of the grid (moving black-hole excision).

In a generalized harmonic gauge [38], the coordinates $x^{\mu}=\left(t, x^{i}\right)=(t, x, y, z$,$) satisfy$

$$
-\nabla_{a} \nabla^{a} x^{\mu}=\Gamma^{\mu}=F^{\mu}
$$

where

$$
\Gamma^{\mu}:=g^{\rho \sigma} \Gamma_{\rho \sigma}^{\mu}=-\frac{1}{\sqrt{-g}} \partial_{\nu} \tilde{g}^{\mu \nu}
$$

with gauge source functions $F^{\mu}\left(x^{\rho}, g^{\rho \sigma}\right)$ (which may depend on the spacetime coordinates and the metric) and with the densitized 4-metric

$$
\tilde{g}^{\mu \nu}:=\sqrt{-g} g^{\mu \nu}
$$

playing the role of the basic evolution variable. In this harmonic formulation, the constraints reduce to the gauge condition

$$
C^{\mu}:=\Gamma^{\mu}-F^{\mu}=0
$$

and the evolution system is based upon the reduced Einstein tensor

$$
E^{\mu \nu}:=G^{\mu \nu}-\nabla^{(\mu} \Gamma^{\nu)}+\frac{1}{2} g^{\mu \nu} \nabla_{\alpha} \Gamma^{\alpha}
$$

Here $\Gamma^{\nu}$ is treated formally as a vector in constructing the 'covariant' derivative $\nabla^{\mu} \Gamma^{\nu}$. When the constraints (4) are satisfied, this gives rise to a hyperbolic evolution system

$$
E^{\mu \nu}=-\nabla^{(\mu} F^{\nu)}+\frac{1}{2} g^{\mu \nu} \nabla_{\rho} F^{\rho}
$$

Provided the gauge source functions do not depend upon derivatives of the metric, they do not enter the principal part of the system and do not affect its well-posedness or numerical 
stability. The evolution system (6) takes the specific form of quasilinear wave equations

$$
\begin{aligned}
\partial_{\rho}\left(g^{\rho \sigma} \partial_{\sigma} \tilde{g}^{\mu \nu}\right) & -2 \sqrt{-g} g^{\rho \sigma} g^{\tau \lambda} \Gamma_{\rho \tau}^{\mu} \Gamma_{\sigma \lambda}^{\nu}-\sqrt{-g}\left(\partial_{\rho} g^{\rho \sigma}\right)\left(\partial_{\sigma} g^{\mu \nu}\right)+\frac{g^{\rho \sigma}}{\sqrt{-g}}\left(\partial_{\rho} g^{\mu \nu}\right)\left(\partial_{\sigma} g\right) \\
& +\frac{1}{2} g^{\mu \nu}\left(\frac{g^{\rho \sigma}}{2 g \sqrt{-g}}\left(\partial_{\rho} g\right)\left(\partial_{\sigma} g\right)+\sqrt{-g} \Gamma_{\rho \sigma}^{\tau} \partial_{\tau} g^{\rho \sigma}+\frac{1}{\sqrt{-g}}\left(\partial_{\sigma} g\right) \partial_{\rho} g^{\rho \sigma}\right) \\
+ & 2 \sqrt{-g} \nabla^{(\mu} F^{\nu)}-\sqrt{-g} g^{\mu \nu} \nabla_{\rho} F^{\rho}+\sqrt{-g} A^{\mu \nu}=0
\end{aligned}
$$

where we have included the possibility of a constraint-adjustment term

$$
A^{\mu \nu}:=C^{\rho} A_{\rho}^{\mu \nu}\left(x^{\rho}, g_{\rho \sigma}, \partial_{\tau} g_{\rho \sigma}\right),
$$

i.e., a term which vanishes when the constraints are satisfied and which does not affect the principal part of the evolution system.

Note that we do not explicitly enforce the harmonic constraints (4) during the evolution. Instead, we invoke the Bianchi identities which imply wave equations of the homogeneous form

$$
g^{\rho \sigma} \partial_{\rho} \partial_{\sigma} C^{\mu}+L_{\sigma}^{\mu \rho} \partial_{\rho} C^{\sigma}+M_{\sigma}^{\mu} C^{\sigma}=0
$$

where the matrices $\boldsymbol{L}$ and $\boldsymbol{M}$ are functions of the metric and its first and second derivatives.

Given constraint-preserving initial and boundary conditions, the uniqueness of the solutions to (9) guarantees that the harmonic constraints be conserved during the evolution. On the other hand, constraint-preserving initial data also require that the initial Cauchy data satisfy the standard Hamiltonian and momentum constraints. Also, since the harmonic constraints imply evolution equations for the lapse and shift, the only remaining free initial data in addition to the usual Cauchy data (the 3-metric and extrinsic curvature of the Cauchy hypersurface) are the initial choices of lapse and shift and of the gauge source functions. Note that an initial choice of the gauge source functions is effectively equivalent to a choice in the initial evolution of the lapse and shift.

\subsection{Constraint adjustment and damping}

The constraint adjustments implemented in the code are those investigated by Babiuc et al [25] and have the general form

$$
A^{\mu \nu}:=-\frac{a_{1}}{\sqrt{-g}} C^{\rho} \partial_{\rho} \tilde{g}^{\mu \nu}+\frac{a_{2} C^{\rho} \nabla_{\rho} t}{\varepsilon+e_{\sigma \tau} C^{\sigma} C^{\tau}} C^{\mu} C^{\nu}-\frac{a_{3}}{\sqrt{-g^{t t}}} C^{(\mu} \nabla^{\nu)} t,
$$

where the $a_{i}>0$ are adjustable parameters (in the runs reported here we have set $a_{i}=1$ ), $e_{\sigma \tau}$ is the natural metric of signature (++++) associated with the Cauchy slicing and $\varepsilon$ is a small positive number chosen to ensure numerical regularity. The effects of these adjustments in suppressing long wavelength instabilities in standardized tests for periodic boundary conditions have been discussed by Babiuc et al [25].

In particular, the first and second terms in the adjustments (10) have been shown to be effective in suppressing constraint-violating nonlinear instabilities in shifted gauge-wave tests. The third term in (10), on the other hand, was first considered in [39] and leads to constraint damping in the linear regime. Although it has been used effectively by Pretorius $[11,27]$ in black-hole simulations, it was not effective in the nonlinear regime of the shifted gauge-wave test [25].

We also note that the work reported in [25] has shown that adjustments which scale quadratically with $C^{\mu}$ (or with higher powers) take effect too late to counter the growth of a constraint-violating instability; and this has led to the specific form for the denominator of the second term in (10). 


\subsection{Gauge conditions}

As noted earlier, the gauge source functions $F^{\mu}$ may be chosen to be arbitrary functions of the spacetime coordinates and metric. They can be viewed as differential gauge conditions on the densitized metric. This serves two important purposes. Firstly, it allows for convergence tests based upon a known spacetime, whose analytic metric $g_{(0)}^{\mu \nu}\left(x^{\rho}\right)$ is specified in a non-harmonic gauge, by choosing

$$
F^{\mu}=-\frac{1}{\sqrt{-g_{(0)}}} \partial_{\nu} \tilde{g}_{(0)}^{\mu \nu} .
$$

Using these analytic gauge source functions, in combination with initial and boundary data consistent with the analytic solution, gives rise to numerically evolved spacetimes that are identical to the analytic solution up to discretization error. This is how the convergence tests reported here have been carried out for the Schwarzschild spacetime expressed in (non-harmonic) Kerr-Schild coordinates. Secondly, and most importantly, the gauge source functions can be used to avoid gauge pathologies.

A major restriction for the form of the gauge source functions is that they cannot depend on the derivatives of the metric. In particular, they cannot depend on the location or shape of the MOTS and this is a problem when moving black holes are present, and where it is important for the coordinates to be able to 'respond' to the black hole motion. In our simulation of binary black holes, we have used the gauge source function

$$
F^{\mu}=\frac{\omega}{\sqrt{-g}}\left(\tilde{g}^{t \mu}-\eta^{t \mu}\right)
$$

where $\eta^{\mu \nu}$ is the Minkowski metric and where $\omega=\omega\left(x^{i}\right)$ is a smooth, spherically symmetric, time-independent weighting function with $\omega=1$ over most of the computational domain, but with $\omega=0$ in some neighbourhood of the outer boundary. When spatial derivatives are neglected and $\omega=1$, the resulting gauge condition takes the simpler form

$$
\partial_{t} \tilde{g}^{t \mu}=-\left(\tilde{g}^{t \mu}-\eta^{t \nu}\right),
$$

showing that it forces the densitized lapse and shift to relax to their Minkowski values.

In our first attempts at binary black hole simulations, we have found that this choice of gauge source function keeps the lapse and shift under reasonable control. Similar choices of gauge source functions have been used with success in other binary black hole simulations $[11,27]$.

\subsection{Boundary conditions}

Our evolution domain has a timelike outer boundary and a smooth, spacelike excision boundary inside each MOTS. The harmonic evolution system, in the second-order form (7), consists of quasilinear wave equations whose characteristics are identical to the null directions determined by the metric. As a result, all characteristics leave the spacelike excision boundaries and no boundary conditions are necessary (or allowed).

At the timelike outer boundary, any dissipative boundary condition for the wave equation with shift leads to a well-posed initial-boundary value problem (IBVP). Such dissipative boundary conditions were first worked out in the one-dimensional (1D) case [40-42] and general results for the 3D case have been discussed recently in [23, 43]. For a boundary with normal in the $+x$ direction, such dissipative boundary conditions have the form

$$
\left[(1-\kappa) \partial_{t}+\kappa g^{z \rho} \partial_{\rho}\right] \tilde{g}^{\mu \nu}=q^{\mu \nu}, \quad 0 \leqslant \kappa \leqslant 1,
$$


for each component $\tilde{g}^{\mu \nu}$, where $q^{\mu \nu}$ are the boundary data. The choice $\kappa=0$ gives a Dirichlet condition and $\kappa=1$ gives a Neumann condition. Dirichlet and Neumann conditions are marginally dissipative in the sense that they are purely reflective for modes with $q^{\mu \nu}=0$. A strictly dissipative Sommerfeld-type condition arises when $\kappa$ is chosen so that the derivative on the left-hand side of (14) lies in the outgoing null direction.

In order for the IBVP to be constraint preserving, the boundary data $q^{\mu \nu}$ must be assigned to enforce a homogeneous, dissipative boundary condition on the constraints $C^{\mu}$. Then, with proper initialization, the uniqueness of solutions to equations (9) ensures that the constraints are satisfied throughout the evolution. The first proposal for such constraint-preserving boundary conditions for the harmonic system consisted of a combination of three Dirichlet and seven Neumann conditions on the components of $\tilde{g}^{\mu \nu}$ [22]. However, numerical studies [23] showed that these Dirichlet-Neumann boundary conditions were effective in carrying the signal off the grid but that their marginally dissipative nature reflected the noise and gave poor results in highly nonlinear tests.

The first example of strictly dissipative constraint-preserving boundary conditions which would in principle allow numerical error to leave the grid, was given for a tetrad formulation of the Einstein equations by Friedrich and Nagy [44]. Constraint-preserving boundary conditions of the Sommerfeld type which lead to a well-posed IBVP for the nonlinear harmonic formulation have subsequently been formulated [45]. These Sommerfeld-type boundary conditions have been incorporated in a numerical code which gives vastly superior performance in nonlinear test problems than the Dirichlet-Neumann scheme [24]. However, we have not yet implemented these conditions here; instead we have used a naive version of Sommerfeld boundary conditions which not only is not constraint-preserving but whose numerical implementation is only second-order accurate. In addition, as explained further in section 3, the code uses summation-by-parts (SBP) difference operators which are fourthorder accurate in the interior but only second-order accurate in the vicinity of the outer boundary.

\subsection{Reduction to first-order in time}

In contrast to what is done in $[11,27]$, where the harmonic evolution system is second-order in time, we find it convenient to discretize and use the method of lines to time-integrate an evolution system which is first-order in time. We note that the reduction to first-order in time can be done in a number of ways, some of which may have very different stability properties when discretized (see appendix for a discussion).

Here, we introduce the auxiliary variables

$$
\hat{Q}^{\mu v}:=g^{t t} \partial_{t} \tilde{g}^{\mu \nu}+w g^{t i} \partial_{i} \tilde{g}^{\mu \nu},
$$

where $w=w\left(x^{i}\right)$ is a smooth weighting function with $w=1$ over most of the computational domain but with $w=0$ in a neighbourhood of the outer boundary. We rewrite definition (15) to obtain the time derivatives of $\tilde{g}^{\mu \nu}$ in terms of $\hat{Q}^{\mu \nu}$ and spatial derivatives of $\tilde{g}^{\mu \nu}$

$$
\partial_{t} \tilde{g}^{\mu \nu}=\frac{1}{g^{t t}}\left(\hat{Q}^{\mu \nu}-w g^{t i} \partial_{i} \tilde{g}^{\mu \nu}\right)
$$

Next, we use identity (16) to re-express the principal part of the harmonic evolution equations (7) as

$$
\partial_{\rho}\left(g^{\rho \sigma} \partial_{\sigma} \tilde{g}^{\mu \nu}\right)=\partial_{t} \hat{Q}^{\mu \nu}+(1-w) \partial_{t}\left(g^{t i} \partial_{i} \tilde{g}^{\mu v}\right)+\partial_{i}\left(g^{i t} \partial_{t} \tilde{g}^{\mu v}\right)+\partial_{i}\left(g^{i j} \partial_{j} \tilde{g}^{\mu \nu}\right),
$$

and after using (16) to convert all time derivatives of $\tilde{g}^{\mu v}$ in (17) and (7) into spatial derivatives, we obtain an equation of the form

$$
\partial_{t} \hat{Q}^{\mu \nu}=F^{\mu \nu}\left(\tilde{g}, \partial_{i} \tilde{g}, \partial_{i j} \tilde{g}, \hat{Q}, \partial_{i} \hat{Q}\right) .
$$


Equations (16) and (18) represent our basic evolution equations for the field variables $\tilde{g}^{\mu \nu}$ and $\hat{Q}^{\mu \nu}$, respectively.

\section{Numerical implementation}

\subsection{Finite-difference algorithms}

The code solves the finite-difference equations on a Cartesian grid with finest resolution $\Delta x^{i}=h$, using a cubic outer boundary and with excision boundaries for each black hole. Vertex-centred mesh refinement is applied using the Carpet driver [35], within the framework of the Cactus computational toolkit [36]. The time evolution is carried out by the method of lines using a fourth-order Runge-Kutta scheme, with a fifth-order spatial prolongation and a second-order time interpolation to provide fine-grid boundary data at mesh-refinement boundaries.

While the bulk of the code uses fourth-order accurate centred difference operators to approximate spatial derivatives, in a neighbourhood of the outer boundary we approximate the spatial derivatives by diagonal norm SBP difference operators of fourth-order interior accuracy and of second-order accuracy at the boundary. More specifically, on a grid $x_{I}=x_{0}+\mathrm{i} h$ with boundary at $x_{0}$, these operators, as described by Mattsson and Nordström [46], are

$$
\begin{aligned}
\left(\partial_{x} f\right)_{i=1} & \rightarrow \frac{1}{h}\left(\frac{1}{2} f_{[2]}-\frac{1}{2} f_{[0]}\right), \\
\left(\partial_{x} f\right)_{i=2} & \rightarrow \frac{1}{h}\left(-\frac{4}{43} f_{[4]}+\frac{59}{86} f_{[3]}-\frac{59}{86} f_{[1]}+\frac{4}{43} f_{[0]}\right), \\
\left(\partial_{x} f\right)_{i=3} & \rightarrow \frac{1}{h}\left(-\frac{4}{49} f_{[5]}+\frac{32}{49} f_{[4]}-\frac{59}{98} f_{[2]}+\frac{3}{98} f_{[0]}\right),
\end{aligned}
$$

and

$$
\begin{aligned}
& \left(\partial_{x}^{2} f\right)_{i=1} \rightarrow \frac{1}{h^{2}}\left(f_{[2]}-2 f_{[1]}+f_{[0]}\right), \\
& \left(\partial_{x}^{2} f\right)_{i=2} \rightarrow \frac{1}{h^{2}}\left(-\frac{4}{43} f_{[4]}+\frac{59}{43} f_{[3]}-\frac{110}{43} f_{[2]}+\frac{59}{43} f_{[1]}-\frac{4}{43} f_{[0]}\right) \\
& \left(\partial_{x}^{2} f\right)_{i=3} \rightarrow \frac{1}{h^{2}}\left(-\frac{4}{49} f_{[5]}+\frac{64}{49} f_{[4]}-\frac{118}{49} f_{[3]}+\frac{59}{49} f_{[2]}-\frac{1}{49} f_{[0]}\right)
\end{aligned}
$$

For grid points at the outer boundary, on the other hand, all components of $\hat{Q}^{\mu \nu}$ are updated using a flat-spacetime, homogeneous Sommerfeld boundary condition. With the outer boundary located in the weak-field regime, a Sommerfeld condition applied to $\hat{Q}^{\mu \nu}$ is equivalent to setting the Sommerfeld derivative of $\tilde{g}^{\mu \nu}$ to the value of this Sommerfeld derivative at $t=0$, as determined by the initial data. (By implication, our boundary algorithm is compatible with the initial data.) This boundary condition is effective in maintaining numerical stability. However, it is not constraint-preserving and is a prime target for future code improvement.

In the part of the computational domain near the outer boundary where $w=0$, the evolution algorithm for $\hat{Q}$ and $\tilde{g}$ reduces to a fourth-order version of the subluminal evolution algorithm for evolving the wave equation with shift discussed in [43]. This algorithm is known to be unstable in the region where the shift is superluminal (e.g. near the excision boundary). 
In this superluminal region, we set $w=1$ so that the definition of $\hat{Q}$ (cf equation (15)) makes $\hat{Q}$ the derivative of $\tilde{g}$ in the normal direction to the Cauchy hypersurfaces, which stabilizes the algorithm. A more detailed discussion of this subluminal-superluminal blending is discussed in appendix.

Another important ingredient of our code is numerical dissipation. We find this essential in keeping the algorithm stable in the neighbourhood of the excision domain. In addition, this is also helpful in killing off high-frequency noise generated at the mesh-refinement boundaries. In the interior of the grid, numerical dissipation is added at $O\left(h^{5}\right)$ in the form

$$
\partial_{t}^{2} \tilde{g}^{\mu \nu} \rightarrow \partial_{t}^{2} \tilde{g}^{\mu \nu}+\frac{1}{64} h^{5} \sum_{i} \epsilon_{i}\left(D_{+i} D_{-i}\right)^{3} \partial_{t} \tilde{g}^{\mu \nu},
$$

where $D_{ \pm i}$ are the forward and backward difference operators in the $x^{i}$ direction and $\epsilon_{i}$ is a smooth weighting function. In the neighbourhood of a face of the outer boundary with normal in the $x$-direction, we set $\epsilon_{x}=0$ so that the dissipation applies only in the tangential directions. In carrying out convergence tests for a Schwarzschild black hole, we choose $\epsilon_{i}=0.2$ outside the apparent horizon $(\mathrm{AH})$ and $\epsilon_{i}=2$ inside the $\mathrm{AH}$ (except for a transition region). In the two black hole simulations, we choose $\epsilon_{i}=1$ outside the $\mathrm{AH}$ and $\epsilon_{i}=2$ inside the $\mathrm{AH}$.

\subsection{Moving excision}

The excision algorithm is driven by the apparent horizon finder algorithm [33, 34]. Strictly speaking, this algorithm searches for MOTS, regardless of whether these are apparent horizons or not. We recall that MOTS are defined as smooth, compact two-dimensional surfaces whose outgoing normal null geodesics have zero expansion. With respect to a $3+1$ foliation, the apparent horizon is defined as the three-dimensional hypersurface traced out by the outer boundary of the trapped region in each time slice. If sufficiently smooth, the apparent horizon is foliated by MOTS.

A smooth spacelike boundary is used to excise a region inside each MOTS, resulting in a jagged boundary in the Cartesian grid. The excision boundary is chosen to be centred inside the MOTS and scaled in coordinate size to be 0.7 the size of the MOTS in the binary simulations and 0.8 in the Schwarzschild black hole tests.

We keep the same interior evolution stencil near the excision boundary by introducing the necessary ghost points. Because the dissipation operator (25) would require an excessive number of ghost points we replace it with a third-order form $h^{3}\left(D_{+i} D_{-i}\right)^{2}$ near the excision boundary. Values at the required ghost points are supplied by an extrapolation scheme which was proved to be stable for the case of a boundary aligned with the grid [43]. We have generalized this to the case of a generic smooth boundary in a Cartesian grid following the 'embedded-boundary' method developed by Kreiss and Petersson [47] for formulating a stable Neumann condition. More specifically, we construct a vector $v^{i}$ by taking the flat-space displacement from the centroid of the excised region to the current position, and require that

$$
\sum_{i}\left(v^{i} D_{ \pm i}\right)^{3} \tilde{g}^{\mu \nu}=0 \quad \text { and } \quad \sum_{i}\left(v^{i} D_{ \pm i}\right)^{3} \hat{Q}^{\mu \nu}=0,
$$

where the one-sided differences $D_{ \pm i}$ correspond to the sign of $v^{i}$. The extrapolation condition (26) is applied iteratively at the points near the boundary until the full stencil of ghost points is updated.

Extra care needs to be paid to the identification of ghost points when the excision domain is moving across the grid. In particular, grid points that become interior points at $t^{N}:=t_{0}+N \Delta t$ but were ghost points at $t^{N-1}$ need to be treated as ghost points, i.e., the excision algorithm 
must fill them with extrapolated values during the time integration from $t^{N-1}$ to $t^{N}$. However, when evolving from $t^{N}$ to $t^{N+1}$, these same grid points need no longer be treated as ghost points and can then be labelled as evolution points.

\section{Code tests}

We now present a series of tests to validate our code's stability and accuracy, starting with the evolution of Schwarzschild black holes using static gauge source functions, followed by the head-on collision of two equal-mass black holes and finally the inspiral and merger of the QC-0 initial data set for binary black holes.

\subsection{Single black holes}

We have evolved Schwarzschild black holes for long periods of time to test the stability and accuracy of our evolution algorithm, excision scheme and outer boundary treatment. To study the code's convergence with resolution, we made evolutions with the finest grid spacing set to $h=0.100 M, 0.080 M, 0.0666 M$ and $0.050 M$. Each evolution used a similar grid structure, with five levels of nested 2:1 mesh refinement with the finest grid (spacing $h$ ) extending from the origin to $4 M+9 h$, the next coarsest (spacing $2 h$ ) from the origin to $8 M+9 h$ and so on, up to the coarsest grid (spacing 16h) from the origin to $64 M$. The tests were carried out in octant symmetry. For representative runs, we cross-checked that identical output was produced in full-space simulations.

Each evolution was run for $1000 \mathrm{M}$, with no sign of instability for this full duration. We measure the accuracy of these evolutions by monitoring the apparent horizon's areal radius and the harmonic constraint $C^{0}$. (Convergence results for the error in $\tilde{g}^{00}$ are very similar to those for $C^{0}$.)

Figure 1 shows the error in the apparent horizon areal radius $r_{\mathrm{AH}} / M-2$ for these evolutions. After an initial transient, each evolution displays a slow growth (roughly linear in time) in the apparent horizon. The growth rates are small, and show fourth-order convergence to zero with the resolution. Figure 2, on the other hand, shows the magnitude of $C^{0}$ along the $x$-axis at $t=200 M$. It is clear that while there are regions of excellent pointwise fourthorder convergence, there are also regions where mesh-refinement and other effects make the convergence more problematic.

To gain a clearer picture of the overall convergence behaviour of these evolutions and because the error is by far and large dominated by a small set of grid points near the excision boundary, we use a 'masked' 2-norm $\left\|C^{0}\right\|_{2}$ defined as the root-mean-square norm of $C^{0}$ over only those grid points which are outside the apparent horizon and do not have any finerrefinement-level grid points overlaying them. For example, all of the finest grids outside the apparent horizon would be included in the masked norm, but only that part of the grid with spacing $2 h$ which is outside $4 M+9 h$ would be included, and similarly for the other coarser refinement levels.

After a short initial transient (lasting a time comparable to the outer-boundary crossing time), $\left\|C^{0}\right\|_{2}$ is essentially time-independent in each run. Figure 3 shows the variation of $\left\|C^{0}\right\|_{2}$ with resolution at selected times. As can be seen, the norm decreases with resolution, with a convergence exponent which is always $\geqslant 3$, but varies with resolution. We attribute this behaviour to not having sufficient resolution to see the asymptotic convergence behaviour as introduced by the dissipation operator.

Similar results have also been obtained for spinning black holes, which we have evolved with very high spins. Simulations of Kerr black-holes with spins $J / M^{2}$ up to 0.99 have 


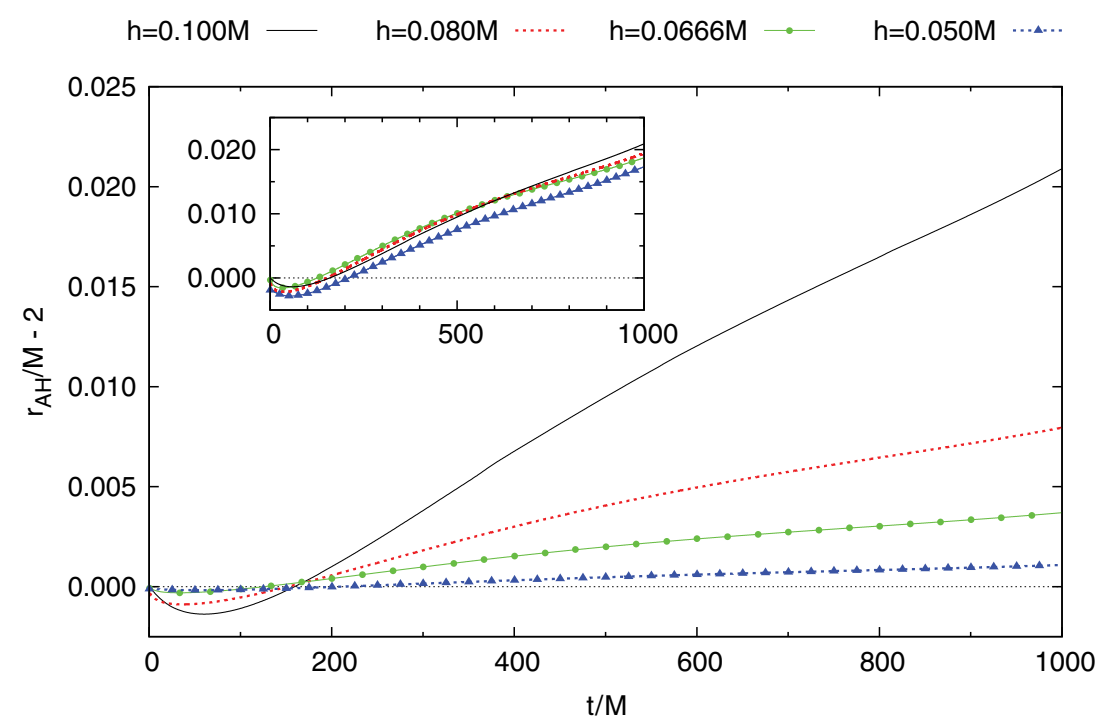

Figure 1. Error $r_{\mathrm{AH}} / M-2$ in the areal radius of the apparent horizon as a function of time for evolutions of Schwarzschild initial data at four different resolutions. The main figure shows $r_{\mathrm{AH}} / M-2$, while the inset shows $(0.1 M / h)^{4} \times\left(r_{\mathrm{AH}} / M-2\right)$, demonstrating that the error shows fourth-order convergence to zero as the resolution is increased.

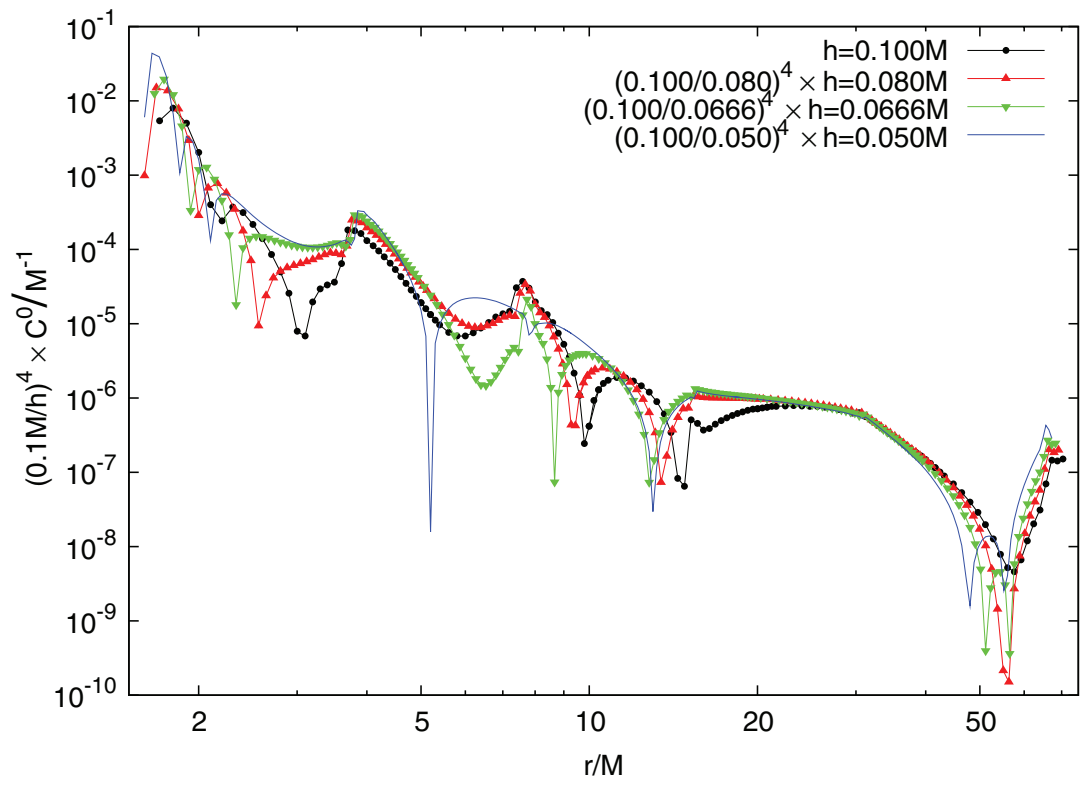

Figure 2. Scaled harmonic constraint $(0.1 M / h)^{4} \times C^{0}$ at $t=200 M$ along the $x$ axis, for evolutions of Schwarzschild initial data at four different resolutions.

shown no apparent signs of instability up to $t=100 \mathrm{M}$. We have not yet investigated them further. 


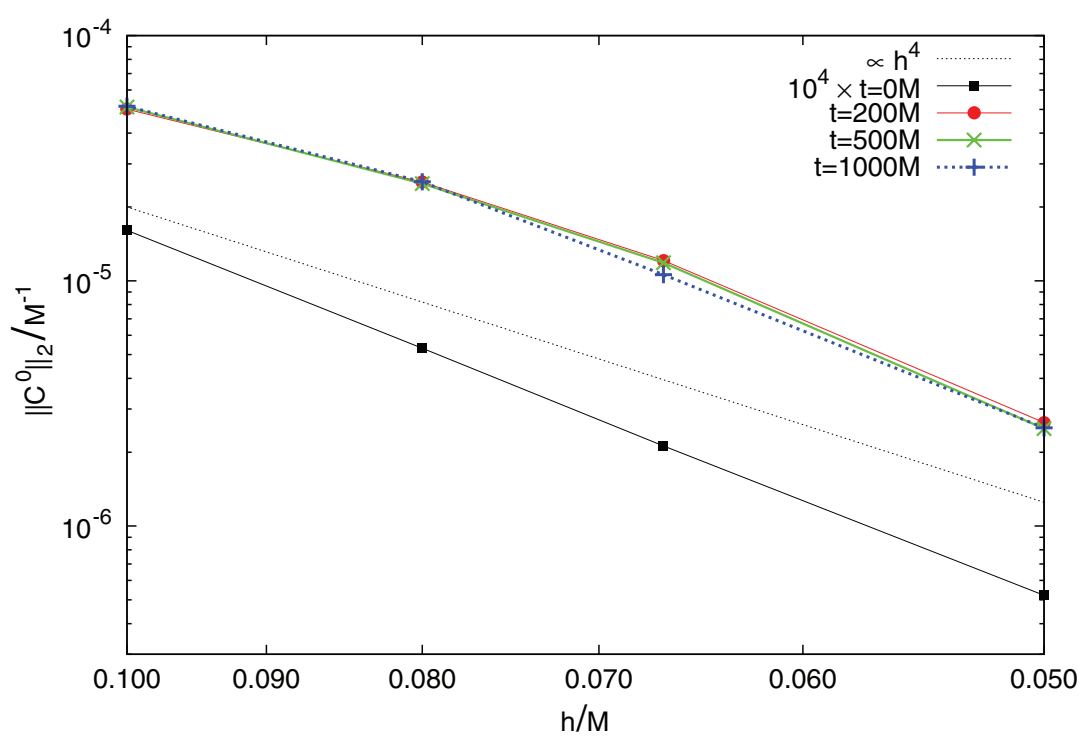

Figure 3. Masked 2-norm of the harmonic constraint $\left\|C^{0}\right\|_{2}$ as a function of the finest grid resolution $h$, at selected times, for evolutions of Schwarzschild initial data at four different resolutions. Note that the $t=200 M, t=500 M$, and $t=1000 M$ curves are almost coincident (because the norms are almost time-independent over this range of times).

\subsection{Binary black hole mergers}

4.2.1. Head-on collision. The binary black hole collision we have carried out is an equalmass, non-spinning head-on collision of two Brill-Lindquist black holes of masses 0.5 each, located on the $z$-axis at $z= \pm 1.16$.

The test was carried out in octant symmetry, using a pure harmonic gauge (i.e., $F^{\mu}=0$ ). The numerical grid was set up using nine levels of refinement, with grid step $h^{(n)}=3.2 \times 2^{-n}, n=0, \ldots, 8$ giving a grid step of $h=0.0125$ on the finest refinement level and an outer boundary at $144 \mathrm{M}$. The chosen grid setup is such that the initial black holes are contained within the bounding box of the finest grid and hence no motion of the finer grids is needed.

After the merger, when the final horizon has an ellipsoidal shape with maximum and minimum radii in a ratio $r_{\min } / r_{\max } \gtrsim 0.6$, the finest refinement level is dropped. This is justified by the fact that the coordinate radius of the final apparent horizon radius is more than double that of the two individual MOTS. Overall, the simulation shows no sign of instability and figure 4 shows the $\ell=2, m=2$, even-parity multipole of the Zerilli function $Q_{20}^{+}$as extracted at $R=60 M$, indicating a well-captured quasi-normal ringing of the black hole.

4.2.2. QC-0 inspiral. We next consider the evolution of the QC-0 initial data defined by [48] and constructed with the highly-accurate spectral elliptic solver of [49]. In contrast with what was done for the head-on collision, we here use the gauge source function (12) in order to keep the lapse from collapsing and the shift from forming large coordinate distortions in the strong-field region. While helping in terms of stability, these gauge source functions also lead to a coordinate growth of the MOTS.

The simulation started out with nine levels of mesh refinements, with the spacing on the individual refinement levels being $h^{(n)}=2.048 \times 2^{-n}, n=0, \ldots, 8$ and yielding a resolution 


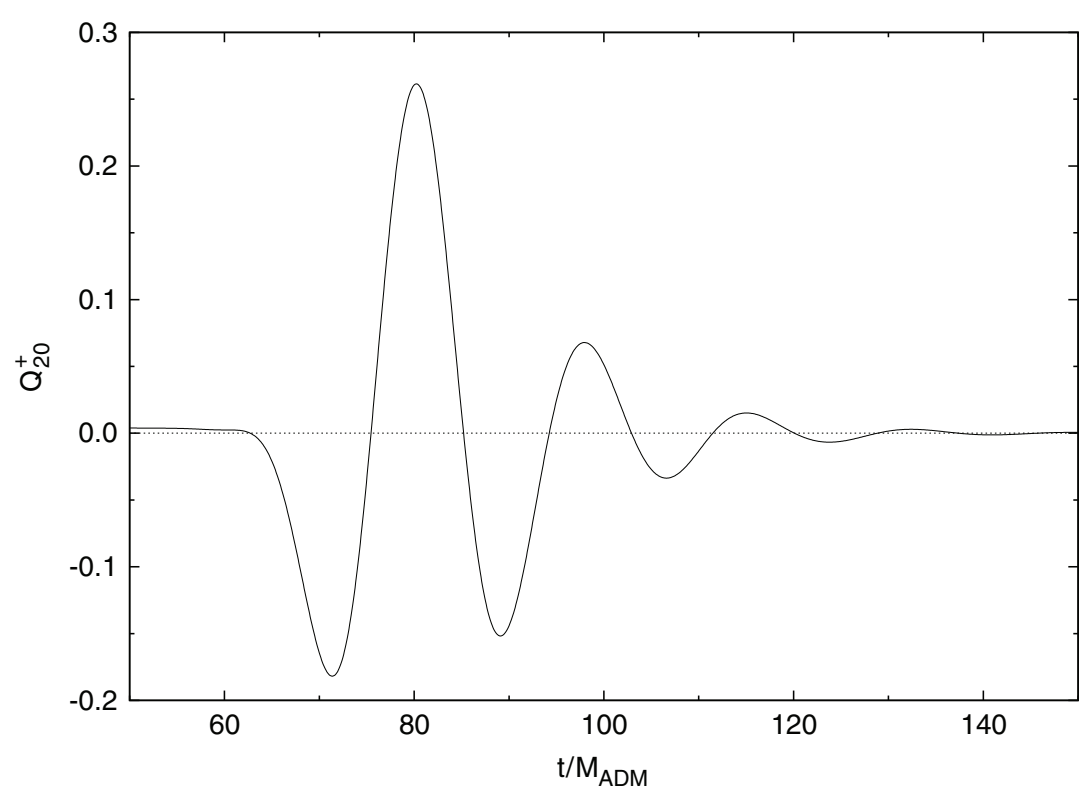

Figure 4. Zerilli waveform for the head-on problem, extracted at $R=60 M$. The outer boundary in this test was at $L=144 M$.

of $0.008 M$ on the finest refinement level, with an outer boundary at $\approx 160 M$. The simulation was carried out in the $x \geqslant 0, z \geqslant 0$ quadrant, taking advantage of the reflection symmetry across $z=0$ and the $\pi / 2$ rotation symmetry around the $z$-axis. Around each black hole we have used a set of refinement levels of size $L^{(n)}=R_{\mathrm{AH}}(t=0) \times 2^{(9-n)}$ so that the finest grid $(n=8)$ would initially be twice the size of the apparent horizon. This refinement structure follows the motion of the black holes across the grid and, as the coordinate growth of the MOTS takes place, we adjust our grid structure by first dropping the finest and later the second finest refinement level. After dropping the second finest level, we re-adjust our grid structure by setting $L^{(n)}=R_{\mathrm{AH}}(t) \times 2^{(7-n)}, n=0, \ldots, 6$, keeping the ratio $L^{(n)} / R_{\mathrm{AH}}(t)$ constant as the MOTS grows further.

At the time of the formation of the common apparent horizon the finest grid has $h^{(6)}=0.032 M$. The individual MOTS were tracked as long as possible. Eventually the horizon finder algorithm hits the excision domain of the other black hole, at which time the individual MOTS are lost.

Figures 5 and 6 reveal an interesting feature of the MOTS dynamics following the formation of a common apparent horizon. The individual MOTS continue to inspiral until they touch (apparently at a single point) and then overlap. Note that, by definition, at the time of touch (and of later intersection) of the individual MOTS, there already exists a third, common MOTS, which is now the apparent horizon. We measure a coordinate velocity of $v \approx 0.16$ of the MOTS as they approach each other. This phenomenon, including the approximate value of $v$, was reproduced using a variety of numerical parameters, including varying resolutions, regridding rules and outer boundary locations. Finer grids allow longer tracking by the apparent horizon finder, which in turn leads to larger overlaps between the individual horizons. The figures we present here were obtained with our best-resolved run.

We have measured the convergence of our QC-0 test by comparing results obtained at three different resolutions. At the time of the overlap of the MOTS, the grid steps were 


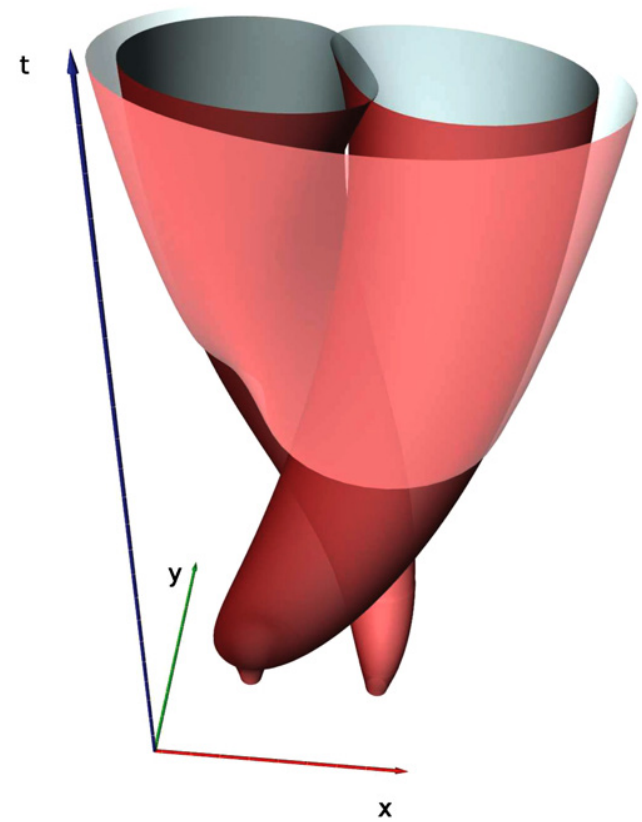

Figure 5. Three-dimensional representation of the QC-0 merger. The axes are $(x, y, t)$. The overlap of the MOTS is clearly visible. Note that these surfaces are actually smooth everywhere; their apparent non-smoothness in some parts of this figure is an artefact of the perspective projection.

$h_{\mathrm{h}}=0.032 M, h_{\mathrm{m}}=0.04 M$ and $h_{1}=0.044 M$. The MOTS touching time for the three resolutions was $T_{\mathrm{h}} \approx 10.73 M, T_{\mathrm{m}} \approx 10.36 M$ and $T_{1}=10.29$. As a first measure of the numerical error, we checked the convergence of $\left\|C^{0}\right\|_{\infty}$ at $t \approx 10.956$. With the excision being a major source of numerical error, the $L_{\infty}$ norm is a reflection of the constraint error near the excised points, including those within the region of overlapping MOTS. Overall, we measure that

$$
\frac{\left\|C_{1}^{0}\right\|_{\infty}}{\left\|C_{\mathrm{h}}^{0}\right\|_{\infty}} \approx\left(\frac{h_{1}}{h_{\mathrm{h}}}\right)^{2.25} .
$$

Such a convergence order can be explained by bearing in mind that the derivatives of the metric contained in the constraint $C^{0}$ are computed using five-point centred stencils. Near the excision boundary, however, two of those five points are updated by extrapolation, using the available three points. This effectively implies that the quantity $C^{0}$ is computed near the excision boundary using a three-point non-centred, first-order derivative stencil, which has an error of $\mathcal{O}\left(h^{2}\right)$.

As an additional measure of the convergence order we have computed the $L_{2}$ norm of the differences between the coordinate shapes $\mathcal{S}$ of the MOTS for the different gridsizes. Given the fact that the three runs had shape information available at different discrete time values, first we interpolated in time, from all three sets of data, to a common set of time slices

$$
t_{n}=t_{\min }+\frac{n-1}{N-1} \times\left(t_{\max }-t_{\min }\right), \quad n=1, \ldots, N .
$$

The value of $N$ was not critical in this convergence test, given the smooth behaviour of the surfaces. We used $N=1000$ for the individual MOTS and $N=100$ for the common apparent horizon. 


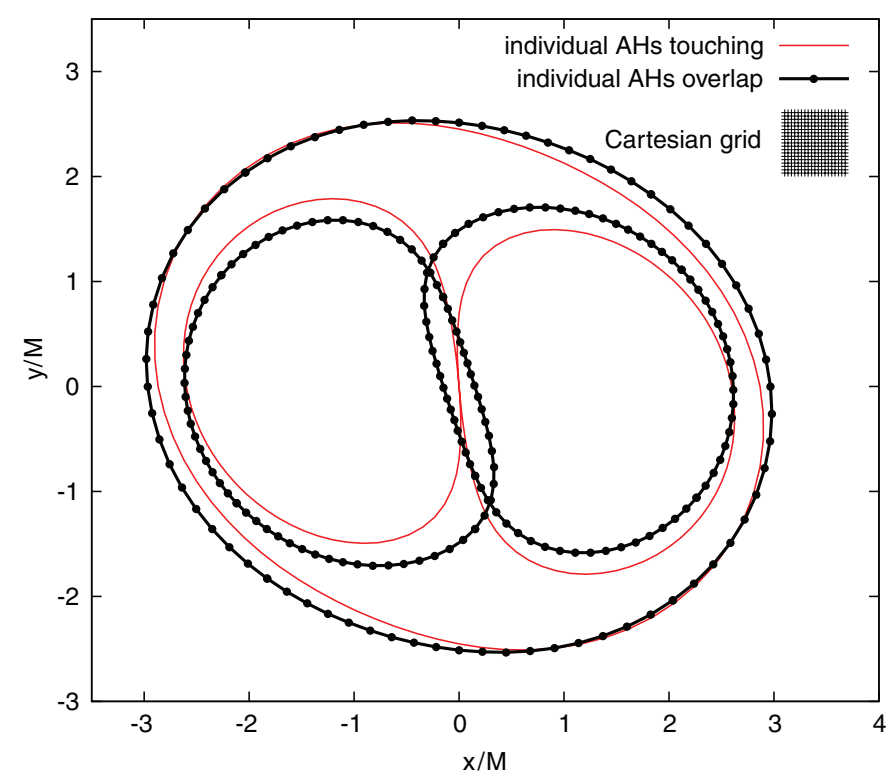

Figure 6. Intersection of the MOTS with the $z=0$ plane at the time when the individual MOTS first touch $(t \approx 10.73 M)$ and just before the individual MOTS are lost $(t \approx 11.47 M)$; for the latter time the individual horizon-finder angular grid points are also shown. At the times shown here the finest grid has $h^{(6)}=0.032 M$; this is illustrated in the plot legend. Note that the MOTSs are well-resolved on both the Cartesian and angular grids at all times, and that they clearly overlap at the latter time.

At each time level $t_{n}$, for each resolution, we had a set of grid points labelled by their angular grid index $[I, J]$ as well as Cartesian coordinates. (Note that for convenience we used the same angular grid resolution of the horizon-finder algorithm for all our runs.) For a given choice of $t_{\min }, t_{\max }$ and $N$ we define the $L_{2}$ norm of the error in the shape of the MOTS to be

$$
\left\|\mathcal{S}_{l}-\mathcal{S}_{h}\right\|_{2}=\sqrt{\sum_{I, J, n} \sum_{i=1}^{3}\left[\left(x_{l}^{i}\left(t_{n}, I, J\right)-x_{h}^{i}\left(t_{n}, I, J\right)\right]^{2} /\left(N_{I} N_{J} N\right)\right.} .
$$

We set $t_{\max }=11.0$ based on the maximum physical time for which horizon data were available for the coarsest run. The convergence rate for the individual MOTS (with $t_{\min }=0$ ) was

$$
\frac{\left\|\mathcal{S}_{l}-\mathcal{S}_{h}\right\|_{2}}{\left\|\mathcal{S}_{m}-\mathcal{S}_{h}\right\|_{2}} \approx\left(\frac{h_{1}}{h_{\mathrm{m}}}\right)^{2.42}
$$

The accuracy of the touching and overlapping MOTS was measured by restricting the $L_{2}$ norm to $t \geqslant t_{\min }=10.4$. The measured convergence rate is $\mathcal{O}\left(h^{2.59}\right)$, while the common (apparent) horizon shape was convergent to $\mathcal{O}\left(h^{2.57}\right)$.

Overall the accuracy of our QC-0 test is between second- and third-order, which is lower than what was measured for the case of an isolated Schwarzschild spacetime. As mentioned above, the most likely causes of the larger error are the close proximity of the moving excision algorithm to the apparent horizon finder interpolation stencils and the $\mathcal{O}\left(\mathrm{d} t^{3}\right)$ time interpolation in the mesh refinement algorithm. (Note that, for time-independent test cases such as a Schwarzschild black hole, the time-interpolation error will effectively not be seen due to the small coefficient in front of the corresponding $\mathcal{O}\left(\mathrm{d} t^{3}\right)$ error term.) 
New insight into the peculiar features of apparent horizons has been revealed in recent numerical simulations [50] and some mathematical machinery has been developed to deal with their properties in a rigorous way $[51,52]$. In particular, a recent theorem due to L Andersson and J Metzger [37] requires that an outer common horizon must already exist if two MOTS come into contact. It is reassuring and stimulating that our results are consistent with this theorem.

\section{Conclusions}

Over the last few years substantial progress has been made in developing mathematical theorems which establish the well-posedness of the harmonic initial-boundary value problem and the stability of its finite difference approximations. We have incorporated some of this theory in developing an explicit in time, finite-difference harmonic code and for which we have presented a series of tests assessing its validity and accuracy. Such tests range from the evolution of isolated black holes to the head-on collision of two black holes and over to a binary black hole inspiral and merger. All of them indicate that stable, convergent and accurate solutions of the Einstein equations in fully nonlinear regimes can be carried out. Furthermore, the merger simulations have also revealed that individual MOTS can touch and even intersect. This novel feature in the dynamics of the MOTS was not found before but is consistent with theorems on the properties of MOTS. This finding raises new questions about the mathematical features of MOTS, which we look forward to exploring.

Overall, these initial results are an important indication that the AEI harmonic code is capable of contributing to the physical understanding of binary black holes currently being achieved by numerical simulation. A key assurance of the validity of numerical results for binary black holes is their confirmation by a variety of codes based upon different theoretical formulations and numerical methods.

The work reported here also suggests that there is room for a great deal of improvement in the numerical methods we have adopted. We have already emphasized the need for implementing a constraint-preserving treatment of the outer boundary. In addition, we have carried out very little exploration to optimize the use of constraint adjustments, the blending of the subluminal and superluminal algorithm, the extrapolation scheme at the extraction boundary, mesh refinement, the addition of numerical dissipation, the choice of gauge conditions, etc. It is particularly reassuring that the code remains stable even though a large amount of error is being generated at the jagged excision boundary. It appears that the outflow nature of the boundary, i.e., its spacelike geometry combined with the lightlike direction of all characteristics of the system, leads to an equilibrium between noise generation and its flux out of the grid.

A number of design choices of our code are based on both the experience gained while building the Abigel code and the published work of Pretorius. It is an optimistic sign that our present results could be obtained by using what was in a number of cases our initial choice for the various code details. We regard this as evidence of the fundamental robustness of the harmonic formulation.

\section{Acknowledgments}

We thank Lars Andersson for discussing his results concerning unstable MOTS prior to publication. We also thank Ralf Kähler for his help in producing the spacetime rendering of the MOTS geometry. The numerical results presented in this paper were obtained on a variety 
of computer systems, most notably with the PEYOTE and BelladonnA clusters at the AEI. This work was partially supported by the National Science Foundation under grant PH-0553597 to the University of Pittsburgh and the SFB-TR7 'Gravitationswellenastronomie' of the German DFG .

\section{Appendix. Blended subluminal-superluminal evolution}

The evolution system (7) consists of coupled quasilinear wave equations whose numerical stability is determined by the principal part. By the principle of frozen coefficients [53], the stability analysis can be reduced to a consideration of the wave equation with shift. Although finite difference approximations to the wave equation are a well-studied problem, the complications introduced by a non-zero shift are peculiar to the black hole excision problem. This was first recognized in [54], where it was suggested that the superluminal shift introduced by tracking the excision boundary could be treated by implicit methods.

Subsequent studies established the stability of explicit finite-difference algorithms, with second-order accuracy, for the case of superluminal evolution. This was first achieved for the $1 \mathrm{D}$ wave equation with shift

$$
g^{t t} \partial_{t}^{2} \Phi+2 \partial_{t} \partial_{x} g^{x t} \Phi+g^{x x} \partial_{x}^{2} \Phi=0,
$$

in work by Calabrese [40] and Szilágyi et al [41]. The standard choice of energy for this system,

$$
E^{(t)}=\frac{1}{2} \int \mathrm{d} x\left[\left(-g^{t t}\left(\partial_{t} \Phi\right)^{2}+g^{x x}\left(\partial_{x} \Phi\right)^{2}\right]\right.
$$

gives rise to a norm when the evolution direction $\partial_{t}$ is timelike. In that subluminal case, summation by parts (SBP) can be used to establish stability of the semi-discrete approximation

$$
g^{t t} \partial_{t}^{2} \Phi+2 g^{x t} D_{0} \partial_{t} \Phi+g^{x x} D_{+} D_{-} \Phi=0
$$

where $D_{+}, D_{-}$and $D_{0}$ are, respectively, the standard forward, backward and centred finite difference approximations for $\partial_{x}$. This ensures that the numerical error is controlled by an estimate for the semi-discrete version of the energy norm $E^{(t)}$. For most methods of lines time integrators, e.g. Runge-Kutta, this estimate extends to the fully discretized system. Algorithm (A.3) has been extended to the 3D subluminal case to give a stable SBP boundary treatment [23].

However, algorithm (A.3) is unstable (and cannot be stabilized by Kreiss-Oliger-type dissipation) when the evolution is superluminal, i.e., when the shift is large enough so that $\partial_{t}$ is spacelike and

$$
g^{x x}=h^{x x}+\frac{\left(g^{x t}\right)^{2}}{g^{t t}}<0,
$$

where $h^{x x}>0$ is the inverse to the spatial metric of the $t=$ const Cauchy hypersurfaces. In that case, when the energy $E^{(t)}$ is no longer a norm, stability can be based upon the positive energy associated with the time-like normal $n^{\mu}$ to the Cauchy hypersurfaces,

$$
E^{(n)}=\frac{1}{2} \int \mathrm{d} x\left[-\frac{1}{g^{t t}}\left(g^{t t} \partial_{t} \Phi+g^{t x} \partial_{x} \Phi\right)^{2}+h^{x x} \partial_{x}^{2} \Phi\right] .
$$

As discussed in the $1 \mathrm{D}$ case $[40,41]$, the discretization

$$
g^{t t}\left(\partial_{t}+\frac{g^{x t}}{g^{t t}} D_{0}\right)^{2} \Phi+h^{x x} D_{+} D_{-} \Phi=0
$$


yields a stable second-order accurate superluminal algorithm. Stable superluminal evolution algorithms for the 3D case have been given by Motamed et al [43], where the global stability of a model black hole excision problem is treated.

Although a stable boundary treatment for the superluminal algorithm (A.6) has been proposed [42], its extended stencil (due to the $D_{0}^{2}$ operator) makes this complicated and an SBP boundary version has not yet been formulated. For this reason we use the $3 \mathrm{D}$ version of the subluminal algorithm (A.3) in the neighbourhood of the outer boundary and blend it with the superluminal algorithm (A.6) by introducing the vector

$$
\hat{n}^{\mu}=\left(g^{t t}, w g^{i t}\right)
$$

and the evolution variable

$$
\hat{Q}=\hat{n}^{\mu} \partial_{\mu} \Phi,
$$

where $w\left(x^{i}\right)$ is a spherically symmetric smooth blending function, with $w=0$ near the outer boundary and $w=1$ (so that $\hat{n}^{\mu}=n^{\mu}$ ) in the interior. It suffices to discuss the frozen coefficient case in which the 1D wave equation (A.1) gives rise to the evolution system for $\hat{Q}$ and $\Phi$,

$$
\begin{aligned}
& g^{t t} \partial_{t} \hat{Q}=-\left(2 g^{x t}-\hat{n}^{x}\right) \partial_{x}\left(Q-\hat{n}^{x} \partial_{x} \Phi\right)-g^{t t} g^{x x} \partial_{x}^{2} \Phi \\
& g^{t t} \partial_{t} \Phi=\hat{Q}-\hat{n}^{x} \partial_{x} \Phi .
\end{aligned}
$$

Note that introduction of the auxiliary variable $\hat{Q}$, which reduces the system to first-order in time, introduces no associated constraints.

For a second-order accurate approximation, we discretize (A.9) according to

$g^{t t} \partial_{t} \hat{Q}=-\left(2 g^{x t}-\hat{n}^{x}\right) D_{0} Q+\left(2 g^{x t}-\hat{n}^{x}\right) \hat{n}^{x} D_{+} D_{-} \Phi-g^{t t} g^{x x} D_{+} D_{-} \Phi$

$g^{t t} \partial_{t} \Phi=\hat{Q}-\hat{n}^{x} D_{0} \Phi$.

In the neighbourhood of the outer boundary, this reduces to the subluminal algorithm (A.3) and in the interior where $w=1$ it reduces to the superluminal algorithm (A.6). The harmonic code uses a fourth-order accurate version of (A.10) in the interior region. An alternative scheme for switching between stable subluminal and superluminal algorithms across the 'artificial horizon' where $\operatorname{det}\left(g^{i j}\right)=0$ is given in [43].

\section{References}

[1] Detweiler S L 1979 Black holes and gravitational waves: perturbation analysis Sources of Gravitational Radiation ed L Smarr (Cambridge: Cambridge University Press) pp 211-30

[2] Hahn S G and Lindquist R W 1964 The two body problem in geometrodynamics Ann. Phys. 29 304-31

[3] Čadež A 1971 Colliding black holes PhD Thesis University of North Carolina at Chapel Hill, Chapel Hill, NC

[4] Eppley K R 1975 The numerical evolution of the collision of two black holes PhD Thesis Princeton University, Princeton, NJ

[5] Smarr L, Čadež A, DeWitt B and Eppley K R 1976 Collision of two black holes: theoretical framework Phys. Rev. D $142443-52$

[6] Smarr L 1977 Spacetimes generated by computers: black holes with gravitational radiation Ann. N Y Acad. Sci. 302 569-604

[7] Smarr L 1979 Gauge conditions, radiation formulae and the two black hole collision Sources of Gravitational Radiation ed L Smarr (Cambridge: Cambridge University Press) p 245

[8] Arnowitt R, Deser S and Misner C W 1962 The dynamics of general relativity Gravitation: An Introduction to Current Research ed L Witten (New York: Wiley) pp 227-65

[9] Frittelli S and Gómez R 2000 Ill-posedness in the Einstein equations J. Math. Phys. 41 5535-49

[10] Cook G B et al 1998 Boosted three-dimensional black-hole evolutions with singularity excision Phys. Rev. Lett. $802512-6$

[11] Pretorius F 2005 Evolution of binary black hole spacetimes Phys. Rev. Lett. 95121101 
[12] Diener P, Herrmann F, Pollney D, Schnetter E, Seidel E, Takahashi R, Thornburg J and Ventrella J 2006 Accurate evolution of orbiting binary black holes Phys. Rev. Lett. 9612110

[13] Campanelli M, Lousto C O, Marronetti P and Zlochower Y 2006 Accurate evolutions of orbiting black-hole binaries without excision Phys. Rev. Lett. 96111101

[14] Baker J G, Centrella J, Choi D-I, Koppitz M and van Meter J 2006 Gravitational wave extraction from an inspiraling configuration of merging black holes Phys. Rev. Lett. 96111102

[15] Gonzáles J A, Sperhake U, Brügmann B, Hannam M and Husa S 2007 Total recoil: the maximum kick from nonspinning black-hole binary inspiral Phys. Rev. Lett. 98091101

[16] Herrmann F, Hinder I, Shoemaker D and Laguna P 2007 Unequal mass binary black hole plunges and gravitational recoil Class. Quantum. Grav. 24533

[17] de Donder T 1921 La Gravifique Einsteinienne (Paris: Gauthiers-Villars)

[18] Fock V 1959 The Theory of Space, Time, and Gravitation (New York: Pergamon)

[19] Choquet-Bruhat Y and Ruggeri T 1983 Hyperbolicity of the 3+1 system of Einstein equations Commun. Math. Phys. 89 269-75

[20] Garfinkle D 2002 Harmonic coordinate method for simulating generic singularities Phys. Rev. D 65044029

[21] Szilágyi B, Schmidt B and Winicour J 2002 Boundary conditions in linearized harmonic gravity Phys. Rev. D 65064015

[22] Szilágyi B and Winicour J 2003 Well-posed initial-boundary evolution in general relativity Phys. Rev. D 68041501

[23] Babiuc M C, Szilágyi B and Winicour J 2006 Harmonic initial-boundary evolution in general relativity Phys. Rev. D 73064017

[24] Babiuc M C and Winicour J 2006 Constraint-preserving Sommerfeld conditions for the harmonic Einstein equations Preprint gr-qc/0612051

[25] Babiuc M C, Szilágyi B and Winicour J 2006 Testing numerical relativity with the shifted gauge wave Class. Quantum Grav. 23 S319-42

[26] Pretorius F 2005 Numerical relativity using a generalized harmonic decomposition Class. Quantum Grav. 22 425-52

[27] Pretorius F 2006 Simulation of binary black hole spacetimes with a harmonic evolution scheme Class. Quantum Grav. 23 S529-52

[28] Lindblom L, Scheel M A, Kidder L E, Owen R and Rinne O 2006 A new generalized harmonic evolution system Class. Quantum Grav. 23 S447-62

[29] Scheel M A, Pfeiffer H P, Lindblom Lee, Kidder L E, Rinne O and Teukolsky S A 2006 Solving Einstein's equations with dual coordinate frames Phys. Rev. D 74104006

[30] Palenzuela C, Olabarrieta I, Lehner L and Liebling S 2006 Head-on collisions of boson stars Preprint gr-qc/0612067

[31] Bona C, Ledvinka T and Palenzuela C 2002 A 3+1 covariant suite of numerical relativity evolution systems Phys. Rev. D 66084013

[32] Bona C, Ledvinka T, Palenzuela-Luque C and Zacek M 2005 Constraint-preserving boundary conditions in the Z4 numerical relativity formalism Class. Quantum Grav. 22 2615-34

[33] Thornburg J 1996 Finding apparent horizons in numerical relativity Phys. Rev. D 54 4899-918

[34] Thornburg J 2004 A fast apparent-horizon finder for 3-dimensional Cartesian grids in numerical relativity Class. Quantum Grav. 21 743-66

[35] Schnetter E, Hawley S H and Hawke I 2004 Evolutions in 3D numerical relativity using fixed mesh refinement Class. Quantum Grav. 21 1465-88

[36] Cactus Computational Toolkit. http://www.cactuscode.org

[37] Andersson L Private communication

[38] Friedrich H 1996 Hyperbolic reductions for Einstein's equations Class. Quantum Grav. 13 1451-69

[39] Gundlach C, Martin-Garcia J M, Calabrese G and Hinder I 2005 Constraint damping in the Z4 formulation and harmonic gauge Class. Quantum Grav. 22 3767-74

[40] Calabrese G 2005 Finite differencing second order systems describing black hole spacetimes Phys. Rev. D 71027501

[41] Szilágyi B, Kreiss H-O and Winicour J 2005 Modeling the black hole excision problem Phys. Rev. D 71104035

[42] Calabrese G and Gundlach C 2005 Discrete boundary treatment for the shifted wave equation Class. Quantum Grav. 23 S343-68

[43] Motamed M, Babiuc M C, Szilágyi B, Kreiss H-O and Winicour J 2006 Finite difference schemes for second order systems describing black holes Phys. Rev. D 73124008

[44] Friedrich H and Nagy G 1999 The initial boundary value problem for Einstein's vacuum field equations Commun. Math. Phys. 201 619-55 
[45] Kreiss H-O and Winicour J 2006 Problems which are well-posed in a generalized sense with applications to the Einstein equations Class. Quantum Grav. 23 S405-20

[46] Mattsson K and Nordström J 2004 Summation by parts operators for finite difference approximations of second derivatives J. Comput. Phys. 199 503-40

[47] Kreiss H-O and Petersson N A 2004 A second order accurate embedded boundary method for the wave equation with Dirichlet data SIAM J. Sci. Comput. 31

[48] Baker J, Campanelli M, Lousto C O and Takahashi R 2002 Modeling gravitational radiation from coalescing binary black holes Phys. Rev. D 65124012

[49] Ansorg M 2005 A double-domain spectral method for black hole excision data Phys. Rev. D 72024018

[50] Schnetter E and Krishnan B 2006 Non-symmetric trapped surfaces in the Schwarzschild and Vaidya spacetimes Phys. Rev. D 73021502

[51] Ashtekar A and Galloway G 2005 Some uniqueness results for dynamical horizons Adv. Theor. Math. Phys. 9 1-30

[52] Andersson L, Mars M and Simon W 2005 Local existence of dynamical and trapping horizons Phys. Rev. Lett. 95111102

[53] Kreiss H O and Lorenz J 1989 Initial-Boundary Value Problems and the Navier-Stokes Equations (New York: Academic)

[54] Alcubierre M and Schutz B 1994 Time-symmetric ADI and causal reconnection: stable numerical techniques for hyperbolic systems on moving grids J. Comput. Phys. 11244 